\title{
Non-immunologic hydrops fetalis and coronavirus disease (COVID-19) - A case report
}

\author{
Faton Krasniqi', Edmond Pistulli², Astrit M. Gashi ${ }^{3}$, Isabere Krasniqii \\ ${ }^{1}$ Department of Neonatology, Faculty of Medicine, University of Pristine, Pristine, Kosovo \\ ${ }^{2}$ Faculty of Technical Medical Sciences, University of Tirana, Albania \\ ${ }^{3}$ Department of Obstetrics and Gynecology, Faculty of Medicine, University of Pristine, Pristine, Kosovo \\ ${ }^{4}$ Department of Family Medicine, Faculty of Medicine, University of Pristine, Pristine, Kosovo
}

\begin{abstract}
Non-immune hydrops fetalis is a severe fetal condition defined as the excessive accumulation of fetal fluid within the fetal extravascular compartments and body cavities. The prevalence of non-immune hydrops fetalis is unknown. Currently, about $90 \%$ of cases of hydrops fetalis are non-immune hydrops fetalis. Non-immune hydrops fetalis causes are multi-factorial. The pathophysiological mechanism of non-immunologic hydrops fetalis is related to abnormal fluid transportation between plasma and tissues. This is due to the increase in hydrostatic capillary pressure and capillary permeability and a reduction of the plasma osmotic pressure or lymphatic flow.

A variety of viral infection agents have been associated with non-immune hydrops fetalis like parvovirus B19, herpes simplex virus, cytomegalovirus, Toxoplasma gondii, Treponema pallidum, but even other viruses that attack the maternal and fetus during pregnancy cannot be excluded as possible causes of non-immune hydrops fetalis. We present one case of non-immune hydrops fetalis who was diagnosed intra-uterine, three weeks after recovery from Coronavirus disease (COVID-19). After much investigation, we could not find any known factor that may be the cause of non-immunologic hydrops fetalis. Therefore, we predisposed a possible causal connection between nonimmunologic hydrops fetalis and Coronavirus disease (COVID-19).
\end{abstract}

Keywords: pregnant women, non-immune hydrops fetalis, coronavirus disease

\section{INTRODUCTION}

Non-immune hydrops fetalis is a severe fetal condition defined as the excessive accumulation of fetal fluid within the fetal extravascular compartments and body cavities, including the serous cavities such as pericardial, pleural, and peritoneal, with associated skin oedema.

The prevalence of non-immune hydrops fetalis is unknown as it is difficult to obtain when many cases are not diagnosed before intrauterine death or may spontaneously resolve antenatal.

Currently, about $90 \%$ of cases of hydrops are nonimmune hydrops fetalis (1), with prevalence reported as 1 in 1,700-3,000 pregnancies (2-4).

Non-immune hydrops fetalis are the result of an increase in interstitial fluid production. The causes can be different, such as those cardiovascular which make up about 21.7\% (as Ebstein malformation, tetralogy of Fallot), hematologic which represent 10.4\% (Hb Bart's HF), chromosomal;13.4\% (Turner syndrome), infectious (parvovirus B19; Toxoplasma gondii; Rubella virus; cytomegalovirus; herpes simplex virus; enterovirus; syphilis; chickenpox virus; Lyme disease), and more rarely: syndromic (Costello syndrome, Meckel syndrome), inborn errors of metabolism (mucopolysaccharidosis, transaldolase deficiency, GM1 gangliosidosis type 1, Niemann-Pick disease type $\mathrm{C}$ ), urinary and thoracic tract malformations, and congenital diaphragmatic hernia or idiopathic (5-8).

Non-immune hydrops fetalis (NIHF) present during the gestational period and can manifest as ascites, pleural or pericardial effusions, also associated with subcutaneous tissue oedema in the fetus. Non-immune hydrops fetalis are often accompanied by poly- 
hydramnios, fetal tachycardia, antenatal hemorrhage, and decreased fetal movements. Mothers may develop massive anasarca, preeclampsia, and eclampsia. Fetal deaths occur from heart failure and hypoxia. Surviving newborns may be present with acute respiratory distress syndrome (ARDS), severe oedema (mainly of abdomen), enlarged liver and spleen and pale skin (9-11).

Diagnosis is usually by ultrasound during the 2 nd to 3rd trimester of gestation, showing fluid accumulation in two or more body cavities and skin oedema. Maternal preeclampsia, polyhydramnios, and decreased fetal movements may lead to suspected nonimmune hydrops fetalis. Laboratory tests such as blood type, Rhesus factor, antibody screening for TORCHES-CLAP, haemoglobin electrophoresis, alpha-fetoprotein tests and maternal anti-SSA/SSB antibodies, as well as Kleihauer-Betke, can also aid in the diagnosis of non-immune hydrops fetalis. Nonimmune hydrops fetalis can enter into differential diagnosis with multiple disorders such as twin-to-twin transfusion syndrome, neonatal hemochromatosis, congestive heart failure, obstructed bowel, fetal abdominal cysts, obstructed urinary system, hepatitis B, hypothrombinemia, hypercalcemia, hypernatremia, and hypothyroidism and diabetes in mother (12-14).

Treatment of non-immune hydrops fetalis depends on the cause. Intrauterine treatment can involve antiarrhythmic drugs (propranolol, digoxin), thoraco-amniotic chest drain, and blood transfusion when anaemia is present. If the non-immune hydrops fetalis is caused by chromosomal abnormalities, the mother may choose to terminate the pregnancy. If the fetus arrives at the term of birth, it should be delivered at a tertiary care centre where the neonate can receive intensive resuscitation procedures (cardiopulmonary resuscitation) in the delivery room, intensive neonatal care, high-frequency ventilation, medications for the kidneys, parenteral nutrition, and removal of excess fluid from of the abdomen and pleural effusion if present $(15,16)$. It is known that a variety of infectious agents have been associated with non-immune hydrops fetalis such as parvovirus B19; Toxoplasma gondii; rubella virus; cytomegalovirus; herpes simplex virus; enterovirus; syphilis; chickenpox virus; Lyme disease, and many other viral agents. Some viral infections about this aetiology are rare, for example; enterovirus infections. We present one case of non-immune hydrops fetalis who was diagnosed intrauterine, three weeks after recovery from coronavirus disease (COVID-19). We predispose a possible association between non-immune hydrops fetalis and coronavirus disease (COVID 19).
Although, the current data for a possible connectivity still does not exist.

\section{CASE PRESENTATION}

The female baby was born preterm (at the 35th week of gestation), weighing $3.3 \mathrm{~kg}$ and height 53 $\mathrm{cm}$, Apgar score $7 / 8$, by vaginal delivery. The mother was 30 years old, multigravida; the two previous children were completely healthy and had no complications during pregnancy or childbirth. Family history is negative for diabetes mellitus, tuberculosis, thromboembolic disease, hypertension, genetic problems, congenital anomalies and any history of congenital heart disease. She denies of cigarette smoking, alcohol, and drug use. Prior prenatal ultrasounds were normal, but an ultrasound done in the 33rd week of gestation had findings indicating fetal ascites and skin oedema (Fig. 1).

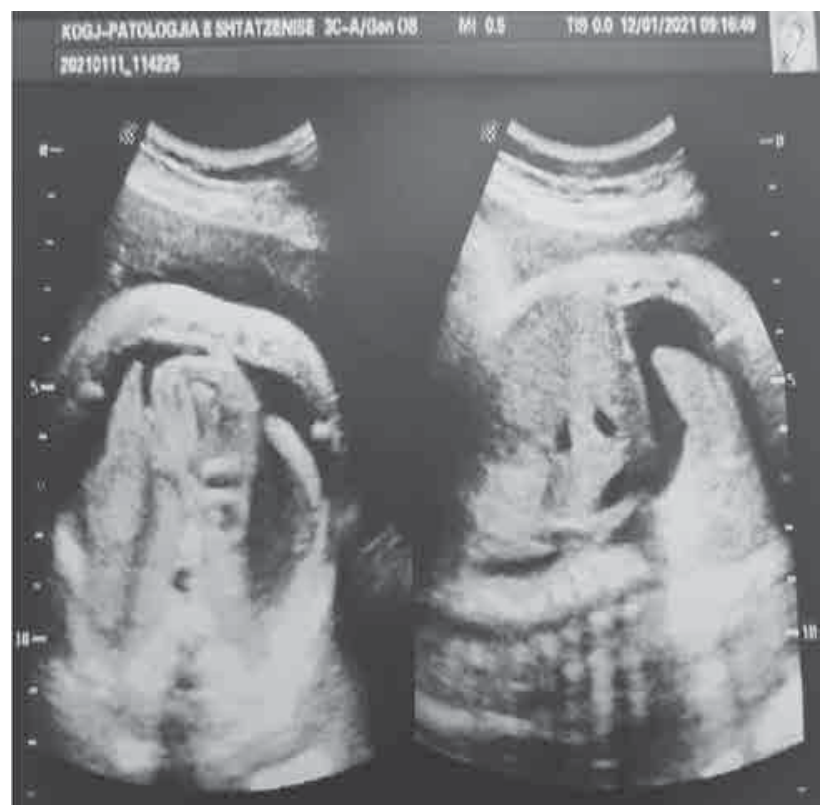

FIGURE 1. Prenatal ultrasound showing fetal ascites

Upon confirming the diagnosis, the mother underwent several multidisciplinary investigations. Laboratory tests such as blood type, Rhesus factor, antibody screening for TORCHES-CLAP, haemoglobin electrophoresis, alpha-fetoprotein tests and maternal anti-SSA/SSB antibodies, as well as Kleihauer-Betke are done and have normal results. Also, karyotype is done and has normal results. Hereditary diseases are also excluded. Upon delivery, the baby had a markedly distended abdomen and mild breathing problems. Also, had oedema of the skin of the body, oedema of the lower and upper limbs, and genital oedema. The patient was transferred to the neonatal intensive care unit for monitoring. Gas analyzes were $\mathrm{pH} 7.35$, pCO2 36.6, pO2 40 mmol/l, HCO3 20.4, BE 5.3, 
oxygen saturation $90 \%$. She was placed in isolation, oxygen mask $31 /$ minute, did not need support for CPAP, hypocalcemia is corrected according to standard protocol. Initial laboratory data were: hemoglobin $17.7 \mathrm{~g} / \mathrm{dl}$, Hct $53.3 \%$, leukocytes $16.5 \mathrm{~mm} 3$ and platelets $184.000 / \mathrm{mm} 3$, total bilirubin $59 \mathrm{mmol} / \mathrm{l}$, indirect bilirubin 5.8, total proteins 38 and albumin 23 . Coombs direct negative. The baby had TORCH screen negative. The patient and mother had the same blood type $\mathrm{O} R$ R-D positive. The X-ray films no show any pathological changes in the lungs, with a normal heart silhouette. An ultrasound study demonstrated moderate ascites in all abdominal quadrants (Fig. 2).

Neonatal ultrasound imaging studies of the brain and the heart were normal. Due to the distension of the abdomen, the pediatric surgeon is consulted, he performed a cleansing enema on the baby and there was no need for paracentesis. Ampicillin and Amikacin were started as initial therapy. Fluid restriction is prescribed and diuretics (furosemide) are given. After confirmation of hypoalbuminemia, albumin human $5 \%$ is prescribed. Abdominal ultrasound is repeated several times and the gradual withdrawal of ascites from the abdomen is observed. Four days after delivery, the patient showed complications such as dyspnea, desaturation crisis and immediately the baby was placed in CPAP for the next 2 days, continues for another two days in an oxygen mask and then in ambient air. The evaluation of enteral food is done, in a minimal beginning, and then it gradually increases until the 10th day, where it passes completely into enteral food. After receiving sterile blood culture, the initial therapy is discontinued on the fifth day of life. An MRI of the abdomen and pelvis is performed on the 24th day of life, which confirms the absence of fluid in the abdominal cavity (Fig. 3).

On the 25th day of hospitalization, she was released home in a generally stable condition and a normal neurological condition. The baby had monthly check-ups, no complications were observed and psychophysical development was normal (Fig. 4).

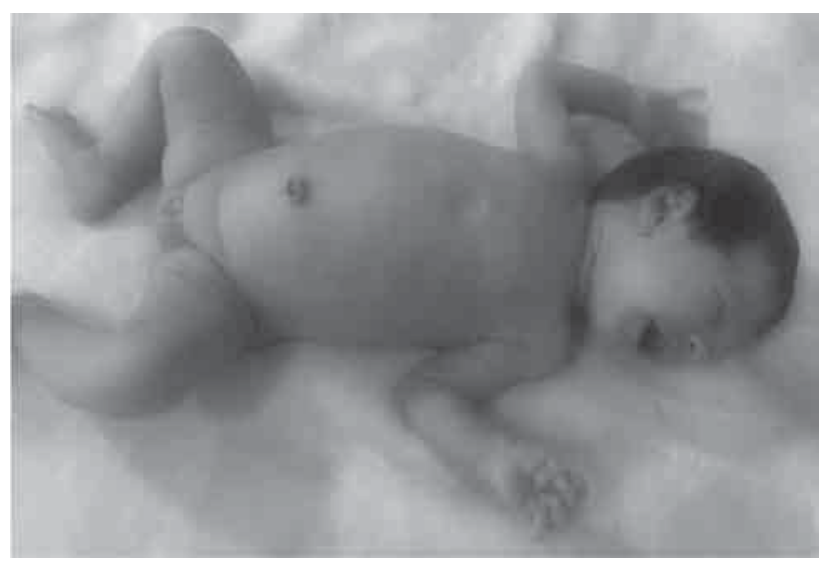

FIGURE 4. The patient on the day of released home from the hospital

\section{DISCUSSIONS}

Hydrops fetalis is excessive fluid accumulation in the subcutaneous tissue in the form of oedema and the body cavities such as ascites, pleural and pericardial effusions. Hydrops fetalis can be mainly catego-

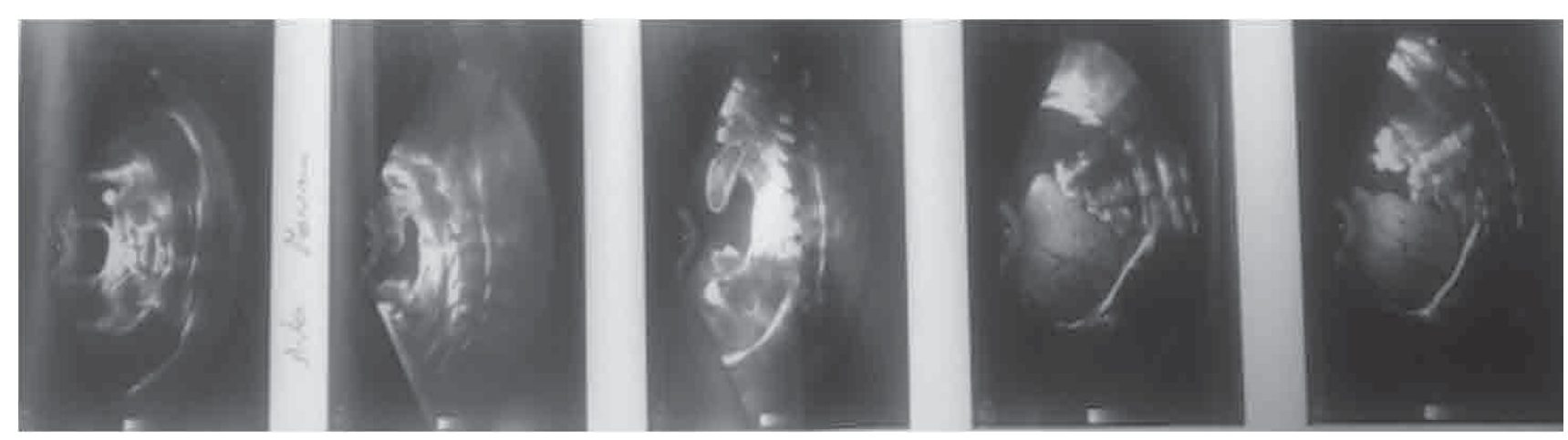

FIGURE 2. Postpartum ultrasound images showing fetal ascites

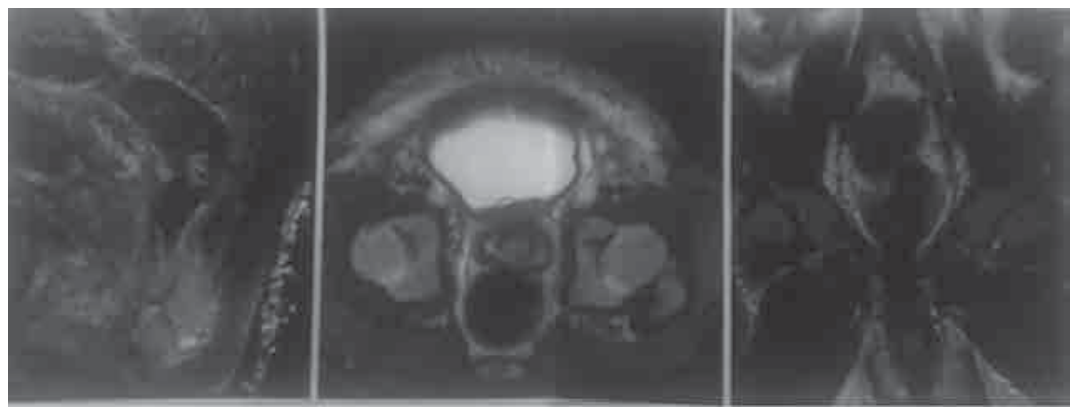

FIGURE 3. MRI views showing the absence of fluid in the intra-abdominal area 
rized as of immune (rhesus isoimmunization) and non-immune causes.

There are a large number of etiological factors that are presumed to be the causes of non-immunologic hydrops fetalis, although in $38 \%$ of cases no possible cause was found (17). One systematic literature review (that has used 24 publications between 2007 and 2013), made 14 classification groups based on the cause of non-immune hydrops fetalis: cardiovascular causes (20.1\%), lymphatic dysplasia (15.0\%), hematologic $(9.3 \%)$, chromosomal $(9.0 \%)$, infections $(7.0 \%)$, syndromic $(5.5 \%)$, TTTF-placental $(4.1 \%)$, miscellaneous $(3.6 \%)$, thoracic $(2.3 \%)$, inborn errors of metabolism $(1.3 \%)$, gastrointestinal $(1.3 \%)$, urinary tract malformations $(0.9 \%)$, extra thoracic tumors $(0.7 \%),(18)$.

The pathophysiological mechanism of non-immunologic hydrops fetalis is related to abnormal fluid transportation between plasma and tissues. The primary causes of the modification of the distribution of body fluids are an increase in hydrostatic capillary pressure and capillary permeability, and a reduction of the plasma osmotic pressure or lymphatic flow (19).

Today, it is known that a variety of agents have been associated with non-immune hydrops fetalis, most notably parvovirus B19, herpes simplex virus, cytomegalovirus, Toxoplasma gondii, Treponema pallidum etc. But other viruses that attack the maternal and fetus during pregnancy cannot be excluded as possible causes of non-immune hydrops fetalis.

We observed a case with non-immunologic hydrops fetalis, which was diagnosed in a pregnant woman three weeks after recovery from coronavirus disease (COVID-19). Signs of fetal hydrops were spotted accidentally during a routine ultrasound examination, at 33 weeks of gestation. In-utero fetal

\section{REFERENCES}

1. Santolaya J, Alley D, Jaffe R, Warsof SI. Antenatal classification of hydrops fetalis. Obstetrics \& Gynecology. 1992 Feb 1;79(2):256-9.

2. Hutchison A, Drew J, Yu V, Williams MI, Fortune D, Beischer N. Non-immunologic hydrops fetalis: a review of 61 cases. Obstetrics \& Gynecology. 1982 Mar 1;59(3):347-52.

3. Machin GA. Hydrops revisited: Literature review of 1,414 cases published in the 1980s. American Journal of Medical Genetics. 1989 Nov;34(3):366-90.

4. Heinonen S, Ryynänen M, Kirkinen P. Etiology and outcome of second trimester non-immunologic fetal hydrops. Acta Obstet Gynecol Scand. 2000 Jan;79(1):15-8.

5. Sileo FG, Kulkarni A, Branescu I, Homfray T, Dempsey E, Mansour S, Thilaganathan B, Bhide A, Khalil A. Non-immune fetal hydrops: etiology and outcomes according to gestational age at diagnosis. Ultrasound in Obstetrics \& Gynecology. 2020 Mar 20.

6. Suwanrath-Kengpol C, Koranantakul O, Suntharasaj T, Leetanaporn R. Etiology and outcome of non-immune hydrops fetalis in southern Thailand. Gynecologic and obstetric investigation. 2005;59(3):134-7. monitoring continues 2 times a week with sonographic studies and is valued in addition to fetal biometry, also Doppler velocimetry of the umbilical artery and middle cerebral artery (MCA). Doppler studies do not indicate in-utero fetal distress or fetal anemia. The fetal biometrics was normal. The patient denies a history of previous cardiac, pulmonary, renal, infectious, genetic, metabolic disease etc. We could not find any known factors that may be the cause of non-immunologic hydrops fetalis. Therefore, we predisposed a possible causal connection between non-immunologic hydrops fetalis and coronavirus disease (COVID-19). Our main goal was to raise a hypothesis for a possible causal connection. Further studies are needed to confirm this.

\section{CONCLUSIONS}

A causal association between coronavirus disease and non-immunologic hydrops fetalis has not yet been confirmed. In the literature, we still do not have any studies or cases that have been reported on this issue. In our case, we excluded all possible etiological factors, known so far for non-immunologic hydrops fetalis and assumed that fetal consequences (in the form of non-immunologic hydrops fetalis) have a strong causal association with coronavirus disease (COVID-19). However, many studies are needed to confirm this. To minimize the long-term consequences for the baby, it is very important to follow-up after the delivery of these cases, with adequate treatment and ongoing care.

\section{Acknowledgement}

The patient has provided informed consent for publication of this case report and accompanying images.

7. Ismail KM, Martin WL, Ghosh S, Whittle MJ, Kilby MD. Etiology and outcome of hydrops fetalis. Journal of maternal-fetal medicine. 2001 Jan 1;10(3):175-81.

8. Bellini C, Hennekam RC, Fulcheri E, Rutigliani M, Morcaldi G, Boccardo F, Bonioli E. Etiology of non-immune hydrops fetalis: a systematic review. American Journal of Medical Genetics Part A. 2009 May;149(5):844-51.

9. Whybra C, Mengel E, Russo A, Bahlmann F, Kampmann C, Beck M, Eich $E$, Mildenberger E. Lysosomal storage disorder in nonimmunological hydrops fetalis (NIHF)-more common than assumed? Report of four cases with transient NIHF and a review of the literature. Orphanet Journal of Rare Diseases. 2012 Dec 1;7(1):86.

10. Chisaka H, Ito K, Niikura H, Sugawara JI, Takano T, Murakami T, Terada Y, Okamura K, Shiroishi H, Sugamura K, Yaegashi N. Clinical manifestations and outcomes of parvovirus B19 infection during pregnancy in Japan. The Tohoku Journal of Experimental Medicine. 2006;209(4):277-83. 
11. Deng Q, Fu F, Yu Q, Li R, Li F, Wang D, Lei T, Yang X, Liao C. Non-immune hydrops fetalis: Genetic analysis and clinical outcome. Prenatal Diagnosis. 2020 Apr 8.

12. Bellini C, Hennekam RC. Non-immune hydrops fetalis: a short review of etiology and pathophysiology. American Journal of Medical Genetics Part A. 2012 Mar;158(3):597-605.

13. Fukushima K, Morokuma S, Fujita Y, Tsukimori K, Satoh S, Ochiai M, Hara T, Taguchi T, Wake N. Short-term and long-term outcomes of 214 cases of non-immune hydrops fetalis. Early Human Development. 2011 Aug 1;87(8):571-5.

14. Moreno CA, Kanazawa T, Barini R, Nomura ML, Andrade KC, Gomes CP, Heinrich JK, Giugliani R, Burin M, Cavalcanti DP. Non-immune hydrops fetalis: a prospective study of 53 cases. American Journal of Medical Genetics Part A. 2013 Dec;161(12):3078-86.

15. Graves GR, Baskett TF. Non-immune hydrops fetalis: antenatal diagnosis and management. American Journal of Obstetrics and Gynecology. 1984 Mar 1;148(5):563-5.

Conflict of interest: none declared Financial support: none declared
16. Nassr AA, Ness A, Hosseinzadeh P, Salmanian B, Espinoza J, Berger V, Werner E, Erfani H, Welty S, Bateni ZH, Shamshirsaz AA. Outcome and treatment of antenatally diagnosed non-immune hydrops fetalis. Fetal Diagnosis and Therapy. 2018;43(2):123-8.

17. Hutchison AA, Drew JH, Yu VY, Williams ML, Fortune DW, Beischer NA. Non-immunologic hydrops fetalis: a review of 61 cases. Obstetrics and Gynecology. 1982 Mar 1;59(3):347-52.

18. Bellini C, Donarini G, Paladini D, Calevo MG, Bellini T, Ramenghi LA, Hennekam RC. Etiology of non-immune hydrops fetalis: an update. American Journal of Medical Genetics Part A. 2015 May; 167(5):1082-8.

19. Bellini C, Hennekam RC. Non-immune hydrops fetalis: a short review of etiology and pathophysiology. American Journal of Medical Genetics Part A. 2012 Mar;158(3):597-605. 